\title{
MUCORMYCOSE OTOCEREBRALE: À PROPOS D'UN CAS
}

\section{OTOCEREBRAL MUCORMYCOSIS : A CASE REPORT}

\author{
L. BOUGHAMOURA, F. HMILA, I. CHABCHOUB, M. ABDELKAFI*, \\ A. TRABELSI ${ }^{* *}$ M. ABROUG, M. YACOUB, K. BOUZOUITA*, AS. ESSOUSSI* \\ SERVICE DE PÉDIATRIE CHU FARHAT HACHED SOUSSE \\ * SERVICE D'ORL CHU FARHAT HACHED SOUSSE \\ **LABORATOIRE D'ANATOMOPATHOLOGIE CHU F HACHED SOUSSE
}

\begin{abstract}
La mucormycose est une affection mycosique rare mais souvent fatale, survenant sur des terrains débilités. Elle est due à la prolifération de champignons cosmopolites de la famille de mucorales. Elle entraîne des lésions délabrantes et extensives des parties molles avec risque de thromboses vasculaires.

Observation : nous rapportons l'observation d'une petite fille, âgée de 3 ans, issue d'un mariage consanguin, hypotrophe, hospitalisée pour otite externe évoluant depuis 3 semaines. La patiente a bénéficié d'une antromastoidectomie et a été mise sous antibiotiques.

Devant une aggravation de l'état local, avec extension de la nécrose et destruction du conduit auditif externe, une infection mycosique est fortement suspectée, sur un terrain particulier de déficit immunitaire probable. L'examen parasitologique d'un prélèvement local a mis en évidence la présence de filaments mycéliens dont la culture a isolé un Rhizopus oryzae, confirmé aussi par l'étude anatomopathologique. L'exploration de l'immunité a mis en évidence un déficit de l'immunitaire céllulaire.Un traitement par amphotéricine B était mal toléré (choc anaphylactique), l'évolution spontanée était rapidement défavorable avec une extension locorégionale importante et une thrombophlébite du sinus latéral homolatéral, aboutissement au décés.

Conclusion : la localisation oto cérebrale de la mucormycose est exeptionnelle, on doit y penser devant une otite externe maligne sur un terrain particulier. La prise en charge doit être urgente afin d'augmenter les chances de survie.
\end{abstract}

Mucormycosis is a relatively rare, potentially life-threatening, fungal infection. It occurs predominately in immunocompromised hosts.

Vascular invasion, thrombosis and rapid ischemic necrosis of infected tissue are the most characteristic features of this pathology.

Early diagnosis and consequently effective treatment are needed to save life in this fatal condition.

Report:

We report a case of otocerebral mucormycosis occurring in an hypotrophic 3-years old girl suffering from language retardation and chronic diarrhea. Immune system's exploration revealed a profound cell-mediated immunity defect. The diagnosis was suspected because of the rapidly extensive necrosis of the external ear conduct, the existence of lateral sinus thrombosis and the resistance to an aggressive antibiotic therapy. The diagnosis was confirmed by identifying Rhizopus oryzae by pathological examination of the necrotic tissue.

Treatment by intravenous amphotericin B was certainly lately begun and unfortunately complicated by anaphylactic shock. Because no alternative drug was possible, such as liposomal amphotericin B, the girl rapidly died.

Conclusion:

Otocerebral mucormycosis is extremely rare.It must be considered when ear lesions are necrotic and rapidly extensive mainly in patients with predisposing conditions. Successful treatment requires tissue excision and early injection of amphotericin B, preferably in its liposomal presentation.

\section{INTRODUCTION}

La mucormycose est une infection fongique rare en pédiatrie, grave, qui survient chez des sujets immunodéprimés ou diabétiques. Elle est due à des champignons opportunistes de la famille de Mucorales [1].

Les auteurs rapportent une observation de mucormycose particulière par sa localisation oto-cérébrale chez une fille âgée de 3ans , chez qui un déficit de l'immunité cellulaire était 
révélé à cette occasion.

Nous rappelons à travers cette observation et une revue de la littérature, les particularités cliniques, para cliniques, évolutives, et thérapeutiques de cette affection très rare en pédiatrie.

\section{OBSERVATION}

Amira, fille âgée de 3ans, hospitalisée pour prise en charge d'une tuméfaction retro-auriculaire droite, évoluant depuis 3 semaines dans un contexte fébrile.

Elle est issue de parents cousins germains, née à terme, avec un poids de naissance à $3,4 \mathrm{Kg}$, un score d'Apgar correct et correctement vaccinée. Elle présente un retard du langage, en fait elle ne prononce aucune syllabe jusqu'à l'âge de 3ans. L'interrogatoire a révélé la notion de diarrhée chronique et des broncho-pneumopathies récidivantes

L'examen physique a montré une fièvre à $38,7^{\circ} \mathrm{C}$, un état général altéré, une pâleur cutanéo-muqueuse, un retard staturo ponderal avec un poids à $9 \mathrm{Kg}$ (- 3,5DS), une taille à 83 cm (-3,5 DS) et un périmètre crânien à 45 cm (-4DS), une tuméfaction rétro auriculaire droite avec des signes inflammatoires locaux. L'auscultation cardio pulmonaire était normale. II n'y avait pas d'hépato splénomégalie. L'otoscopie a mis en évidence un conduit auditif externe droit à paroi inflammé.

Devant ce tableau associant une fièvre avec tuméfaction rétro auriculaire, une mastoïdite a été suspectée survenant sur un terrain particulier de déficit immunitaire probable.

Ce dernier a été évoqué devant la consanguinité, la diarrhée chronique, l'hypotrophie sévère et les antécédents de broncho-pneumopathies récidivantes.

Sur le plan biologique : la numération formule sanguine a objectivé ; GB : 19000 éléments /mm3 (PNN à $89 \%$, lymphocytes à $4 \%$ soit $760 / \mathrm{mm} 3$ ), plaquettes à $160000 / \mathrm{mm} 3$, Hb à 10,4 gr/dl. Cette lymphopénie a persisté ultérieurement sur toutes les numérations faisant évoquer un déficit de l'immunité cellulaire. L'électrophorèse de protides a objectivé une hypo albuminémie (26 gr /l) et une hypo protidémie (47 gr/l) mais pas d'hypo gammaglobulinémie.

Le dosage pondéral des immunoglobulines était normal et la sérologie VIH était négative.

Une étude de l'immunité cellulaire a objectivé une inversion du rapport CD4 / CD8, mais ce déficit n'a pas pu être classé. La patiente a bénéficié d'une mastoïdectomie avec présence d'une inflammation importante et des ulcérations au niveau du conduit auditif externe (CAE) droit qui était le siège d'une fistule à sa paroi postérieure cutanée, avec tissu de granulation abondant du CAE, sans atteinte mastoïdienne.

Devant l'absence d'atteinte mastoïdienne la présence de tissu de granulation au niveau du CAE, le diagnostic de mastoïdite a été redressé et un prélèvement per opératoire a été donc réalisé au niveau du CAE. L'analyse histologique de ce prélèvement a mis en évidence la présence des spores et des filaments mycéliens, larges, ramifiés, non septés et visibles à la coloration de PAS et Grocott (Fig 1 ). Cet aspect a évoqué une mucormycose du CAE.

L'examen mycologique direct était négatif et la culture a isolé un Rhizopus oryzae. Au 5ème jour postopératoire, elle a présenté une paralysie faciale droite.

Sur le plan thérapeutique, elle a été mise d'emblée sous antibiothérapie à large spectre associée à l'traconazole (Triflucan®).

L'amphotéricine $B$ a été débuté, après résultats de l'histologie, à dose test avec une prémédication préalable. La patiente a présenté un choc anaphylactique ayant nécessité son transfert en unité de réanimation pédiatrique et l'arrêt de l'amphotéricine B.

L'évolution ultérieure était défavorable, avec une extension de la lésion nécrotique rétro auriculaire vers la loge parotidienne, objectivée par la tomodensitométrie de l'oreille qui a mis en évidence, un processus pseudo tumoral occupant la loge parotidienne (Fig 2) et s'étendant aux espaces parapharyngées avec lyse de l'os tympanal et des parois du CAE, sans thrombose veineuse cérébrale. Devant cette extension un débridement chirurgical était impossible.

Au bout de 21 jours, la patiente a présenté une fièvre avec altération de l'état général, une somnolence et des vomissements incohercibles. Un scanner cérébral a été réalisé en urgence, à la recherche d'une thrombose veineuse cérébrale. II a confirmé ce diagnostic, en mettant en évidence une thrombose du sinus latéral droit (Fig 3). L'évolution était rapidement défavorable.

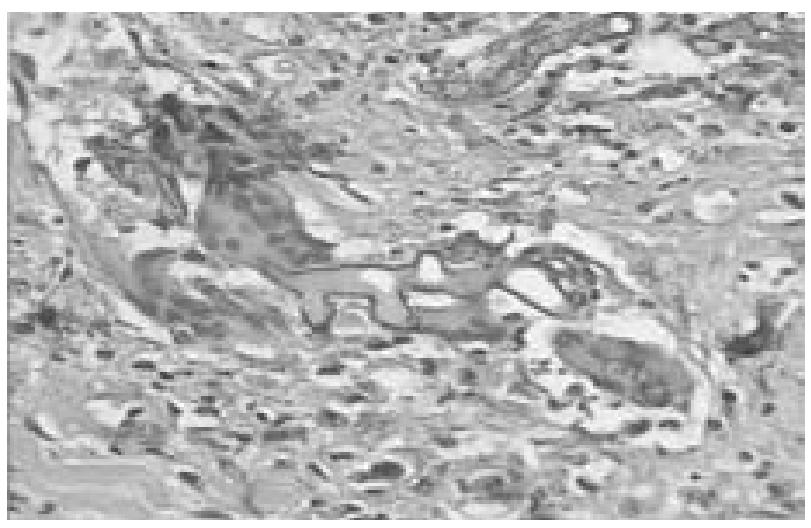

Fig. 1 : Aspect anatomopathologique : Filament mycélien ramifié.



Fig. 2 :1ére TDM : comblement tissulaire de l'oreille externe étendu à l'oreille moyenne. 


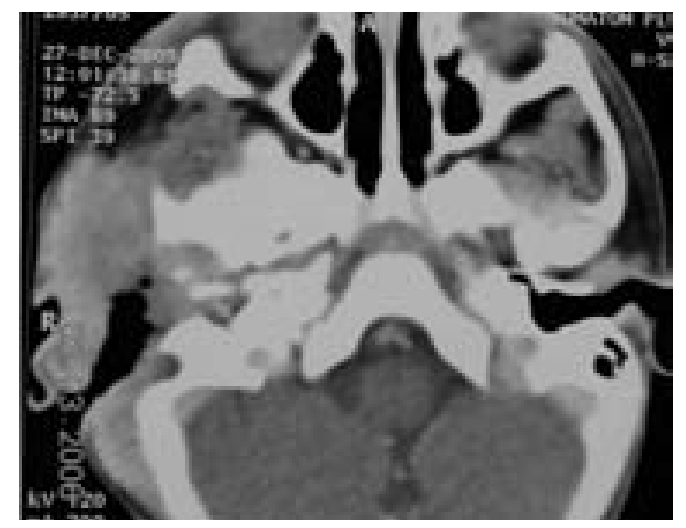

Fig. 3 : 2éme TDM : volumineuse masse de parties molles temporales droites, qui se rehausse intensément en rapport avec un remaniement inflammatoire étendue, thrombophlébite du sinus collatéral droit.

\section{DISCUSSION}

La mucormycose est une infection fongique rare, due à des champignons filamenteux appartenants à la classe de zycomycètes, ordre de Mucorales. Parmi ces derniers, 3 genres sont le plus fréquemment rencontrés : Rhizopus dans $80 \%$ des cas (surtout l'espèce Rhizopus oryzae), Mucor et Absidia [2].

Ces champignons sont ubiquitaires, très répandus dans l'air ambiant, la matière en décomposition, les moisissures et le sol. Leur croissance est extrêmement rapide, mais leur virulence est faible chez les sujets immuno compétents [2]. Ils deviennent pathogènes sur un terrain particulier tel que le diabète en décompensation acido-cétosique, le déficit immunitaire (comme pour notre patiente), les maladies hématologiques entraînant une neutropénie, malnutrition sévère et au cours des traitements corticoïdes ou cytotoxiques[1, 3-6].

Elle a été rapportée chez des nouveaux-nés, souvent prématurés avec un faible poids de naissance, elle est favorisée dans ces situations par la corticothérapie prescrite pour une pathologie respiratoire, l'antibiothérapie à large spectre et les soins intensifs [ 7]. Cependant, elle a été rapportée chez des sujets immunocompétents [8].

Les mécanismes physiopathologiques à l'origine de cette affection, sembleraient impliquer plusieurs facteurs : la neutropénie, un défaut de chémotactisme des polynucléaires neutrophiles et un défaut de la phagocytose. Par ailleurs, l'hyperglycémie altère la fonction phagocytaire et l'acidose métabolique augmente les taux de fer libre non lié à la transferrine.Tous ces facteurs différemment réunis favorisent la croissance fongique [9]. Leur pouvoir pathogène, est basé sur leur grande affinité pour les parois vasculaires, entraînant ainsi la formation des thromboses et nécrose ischémique des tissus. La propagation se fait ensuite sur le plan locorégional ou à distance de façon métastatique $[1,10]$.

Sur le plan clinique, la localisation rhino faciale ou rhino cérébrale est la plus fréquente ( $40 \%$ des cas), suivie de la localisation pulmonaire [5, 10-11].
La localisation cutanée, décrite surtout chez des prématurés, avec souvent une porte d'entrée cutanée favorisée par les pansements occlusifs et particulièrement les cathéters veineux $[7,12]$.

La forme disséminée affecte plusieurs viscères (poumons, foie, tube digestif, ...) [1].

Certaines localisations exceptionnelles étaient rapportées, tel que la localisation musculaire [3].

La localisation oto cérébrale, sans atteinte de la cavité nasale, des sinus paranasaux et de l'orbite, est très rare. En effet, Yun [13] a rapporté un cas de mucormycose de l'oreille moyenne, isolée, compliquée d'une paralysie faciale chez un patient diabétique. Macdonell [4], a rapporté en 1987, l'observation d'un diabétique ayant présenté une mucormycose oto cérébrale, diagnostiquée tardivement et dont l'évolution était défavorable. A l'autopsie,il y avait une atteinte de la parotide avec thrombophlébite du sinus caverneux.

La particularité de notre observation, c'est aussi la localisation d'emblée auriculaire puis parotidienne et cérébrale sans atteinte initiale des sinus maxillaires, cela pourrait être expliquer, par le faite que ces derniers ne sont pas totalement aérés à cet âge (3ans).

Les examens radiologiques (TDM - IRM) cérébraux et du massif facial vont permettre de préciser les images de sinusite, la destruction osseuse et permettent de suivre l'extension locorégionale des lésions vers l'orbite, le cerveau et éventuellement préciser la présence de thromboses cérébrales [1].

Pour notre patiente, le scanner a objectivé l'extension des lésions vers la loge parotidienne et vers le cerveau par la suite.

Le diagnostic de certitude de la mucormycose, quelle que soit sa localisation, repose sur la mise en évidence du champignon. L'examen mycologique direct est rarement positif, et la culture sur milieu de Sabouraud (en 24 à 48 heures) est souvent négative $[1,11]$.

L'examen anatomopathologique du matériel nécrotique, permet de retrouver les filaments mycéliens larges, non septés avec des ramifications à angle droit [1, 10,13].

Le traitement doit être instauré dès la présomption clinique, et après avoir effectué les prélèvements mycologiques. L'amphotéricine B est le traitement de choix [7,14]. Les recommandations actuelles, sont d'utiliser d'emblée les formes liposomales d'amphotéricine $\mathrm{B}$ à des doses élevées $(15 \mathrm{mg} / \mathrm{kg} / \mathrm{j})[5,9,11]$. La durée du traitement antifongique est variable selon l'évolution de 6 semaines à 3 mois.

Une autre alternative thérapeutique a été proposée, basée sur l'association de la caspofungine (Cancidas R) avec un dérivé imidazolé [15].

A coté du traitement antifongique, un débridement chirurgical doit être toujours associé. En effet, la résection chirurgicale des tissus nécrosés permet de réduire la charge fongique et améliore la pénétration des antifongiques dans les zones infectées [9,14].

Chez notre patiente, le choc anaphylactique à l'amphotérici- 
ne $\mathrm{B}$, nous a conduit à arrêter ce traitement et la non disponibilité d'autres alternatives thérapeutiques dans notre pays, étaient des obstacles dans la prise en charge.

Le pronostic de cette pathologie est sombre (85\% de mortalité en cas d'atteinte cérébrale), malgré un traitement médico chirurgical bien conduit $[1,10]$. En l'absence d'atteinte cérébrale le pronostic est meilleur $[5,12]$.

\section{CONCLUSION}

La mucormycose oto cérébrale est une infection fongique rare chez l'enfant, dont il faut savoir y penser devant, une otite externe maligne chez un enfant à risque. L'examen mycolo- gique et/ou histologique d'un prélèvement local permet le diagnostic rapide. Le traitement doit être instaurer en urgence afin d'améliorer le pronostic.

\section{REFERENCES}

1- Battikh R, Labbene I, Ben Abdelhafidh N, Bahri M, Jbali A, Louzir B et al. Mucormycose rhinofaciale : à propos de 3 cas. Med Maladies infect $2003 ; 33: 427-30$

2- Viaud JF, Gin M, Couprie B, Stoll D, Aubertin J. Infections à mucorales chez le patient diabetique. Rev Med Interne 1994: $15: 541-5$.

3- Mookang C, Sik Kim H, Soon Song K, Junglee H, Young Kwon K. Localized muscular mucormycosis in child with acute leukemia. Jpn Clin Oncol 1997; 27(5): 357-60.

4- Macdonell RA, Donnan GA, Kalnins RM, Richards MJ, Bladin PF. Otocerebral mucormycosis - a case report.Clin Exp Neurol 1987; 23: 22532.

5- Chastagner P, Maillot D, Kures L, Georges JL, Schmitt C, Bordigoni P et al.

Evolution favorable d'une mucormycose orbito naso sinusienne compliquant le traitement d'induction d'une leucémie aigue lymphoblastique. Arch Pédiatr 1995; 2(1) :47-51.

6- Gonzalez Nunez I, Dosal Caruso L, Diaz Jidy M, Torres Gomez De Cadiz Silva A, Martinez Machin G. Pulmonary mucormycosis infection in a boy with AIDS. Rev Cubana Med Trop 1997; 49(3):218-21.

7- Scheffler E, Miller GG, Classen DA. Zygomycotic infection of neonatal Upper Extremity.

Journal of Pediatric Surgery 2003; 38(7):16-17.

8- Sridhara S R, Paragache G, Panda NK, Chakrabarti A. Mucormycosis in immunocompetent individuals: an increasing trend.J. Otolaryngol 2005;
34(6):402-6.

9- Spellberg B, Edwards J Jr, Ibrahim A. Novel perspectives on mucormycosis :pathophysiology presentation and management. Clin Microbiol Rev 2005; 18(3): 556-69.

10- Trabelsi A, Soua A, Hachicha L, Fathallah Mili A, Sriha B, Mokni M et al. Mucormycose et diabete : a propos de trois cas. Rev Med Liege 2005 ; $60(56): 545-548$

11- Virchova Z, Beuret P, Boyer M, Chanoz J. Mucormycose pulmonaire fatale chez une patiente diabétique.Ann.Fr.Anesth Réa $2006 ; 25: 40-42$.

12- Morales-Aguirre JJ,Aguero-Echeverria WM,Ornelas-Carsolio ME Resendiz-Sanchez J, Gomez-Barreto D, Cashat-Cruz M.Successful treatment of a primary cutaneous zygomycosis caused by Absidia corymbifera in a premature new born.Pediatr.Infect Dis J 2004;23(5): 470-2.

13- Yun MW, Lui CC, Chen WJ. Facial paralysis secondary to tympanic mucormycosis :case report.Am J Oto $1994 ; 15(3)$ :413-4.

14- Barron MA,Lay M, Madinger NE. Surgery and treatment with highdose liposomal Amphotericin eradication of craniofacial zygomycosis in a patient with disease who had undergone allogenic hematopoietic transplantation.J Clin Microbiol 2005;43(4):2012-14.

15- Nivoix Y, Zamfir A, Lutun P, Kara F, Remy V, Lioure B et al. Combination of Caspofungin and azole or an amphotericin $B$ formulation in invasive fungal infections. J.infect 2006;52(1):67-74. 